\title{
Aggressive Behavior in School-aged Children: Clusters based on Anger, Empathy and Testosterone and Cortisol Measures
}

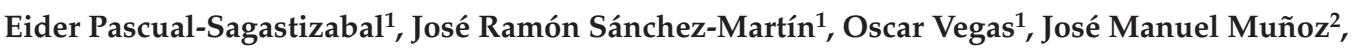 \\ Paloma Braza ${ }^{2}$, María Rosario Carreras ${ }^{2}$, Nora del Puerto-Golzarri ${ }^{1}$ and Aitziber Azurmendi ${ }^{1}$ \\ 1 Universidad del País Vasco (Spain) \\ ${ }^{2}$ Universidad de Cádiz (Spain)
}

\begin{abstract}
The aim of this piece of research was to study the existence of clusters based on anger, empathy and cortisol and testosterone measures associated with aggressive behavior in school-aged children. The sample group comprised 139 eight-year-old children (80 boys and 59 girls). Aggressive behavior was measured using the Direct and Indirect Aggression Scale. Both psychological and biological variables were used to determine psychobiological profiles. The psychological variables considered were trait anger, measured using the State-Trait Anger Expression Inventory for Children and Adolescents, and empathy, measured using the Empathy Quotient-Child Version. Testosterone and cortisol concentrations were measured through saliva samples and analyzed using an ELISA (Enzyme-linked immunosorbent assay). A Cluster Analysis revealed three clusters which were clearly different as regards their psychological and biological characteristics. The analysis of variance (ANOVA) revealed that the cluster characterized by having higher anger levels, lower empathy levels and higher testosterone and cortisol levels was more aggressive than the other two $\left(p<.0001, \eta^{2}=.19\right)$. The results indicate that studying psychological and biological variables together may help establish differentiated aggression patterns among children.
\end{abstract}

Received 14 September 2018; Revised 27 September 2019; Accepted 30 September 2019

Keywords: anger, aggressive behavior, cortisol, empathy, testosterone.

There is widespread consensus regarding the fact that, during very early childhood, it is possible to identify subjects whose trajectories predict a stable use of aggression in the future. This is particularly true in the case of the more concerning manifestations of this behavior, such as physical aggression (Côté, Vaillancourt, Barker, Nagin, \& Tremblay, 2007; Tremblay, Vitaro \& Côté, 2018). Given the stability of aggressive behavior throughout the lifecycle, it is important to study the variables which contribute during childhood to the manifestation of aggressive behavior. There are numerous variables, both psychological and biological, which may be associated with aggressive behavior, and may even predict it. However, some have been the object of more research than others, and the information that is therefore available about them is much more plentiful.

Correspondence concerning this article should be addressed to Eider Pascual Sagastizabal. Universidad del País Vasco. Departamento de Procesos Psicológicos Básicos y su Desarrollo. 20018 San Sebastián (Spain).

E-mail: eider.pascual@ehu.es

This study was supported by a grant provided by the Spanish Ministerio de Ciencia e Innovación (SEJ2007-62897) and funding provided by the Gobierno Vasco (GIC07/19-IT-238-97). We would like to express our gratitude to the teaching staff, parents and children of the participating schools.
Within the large body of research that associates different psychological variables with aggressive behavior, highly consistent results have been found in relation to some variables that either strengthen or attenuate aggression. Anger is one of the main psychological variables considered to increase aggression (Campbell, 2006), with several studies observing a positive association between anger and aggressive behavior (e.g. Daff, Gilbert, \& Daffern, 2015). Anger is a negative emotion that is usually felt in response to threat, disruption of ongoing behavior or the potential infliction of harm by another individual, particularly when said harm is perceived to be unjustified (Campbell, 2006). Anger is a basic emotion which has a number of different components (state anger, trait anger, etc.), although the majority of research into the relationship between anger and aggression has focused exclusively on trait anger, i.e. the chronic tendency to feel and express that emotion (Fung, Gerstein, Chan, \&

How to cite this article:

Pascual-Sagastizabal, E., Sánchez-Martín, J. R., Vegas, O., Muñoz, J. M., Braza, P., Carreras, M. R., ... Azurmendi, A. (2019). Aggressive behavior in school-aged children: Clusters based on anger, empathy and testosterone and cortisol measures. The Spanish Journal of Psychology, 22. e42. Doi:10.1017/sjp.2019.42 
Engebretson, 2015). Anger does not necessarily lead to aggression, although it can predict it, and several studies have found a positive association between this psychological variable and aggressive or externalizing behavior in children, pre-adolescents and adolescents (e.g. Clay, Hagglund, Kashani, \& Frank, 1996).

At the opposite end of the scale is empathy, a variable considered to reduce aggressive behavior (Campbell, 2006). As a general construct, empathy is defined as the ability to perceive and understand other people's emotions and to react to them in an appropriate fashion (Leiberg \& Anders, 2006). It is widely accepted that empathy fosters prosocial behavior (e.g. Warden \& MacKinnon, 2003) and inhibits, or at least mitigates, aggressive or antisocial conduct (Jolliffe \& Farrington, 2006). Individuals who are capable of understanding the negative emotional reaction of others tend to inhibit their own aggressive behavior, or are at least less inclined to continue engaging in this behavior or acting in an antisocial manner in the future (Feshbach, 1975). Diverse studies have found a statistically significant negative relationship between empathy and aggressive behavior (e.g. Berger, Batanova, \& Duncan, 2015).

As regards the biological variables that influence aggression, many studies have focused on the impact of steroid hormones on this behavior. Of all the steroid hormones in our bodies, special attention has been paid to testosterone and cortisol. In general, the majority of studies have observed a positive association between aggressive behavior and testosterone levels, both in adults (Archer, 2006, 2009) and in children (Barzman et al., 2013; Sánchez-Martín et al., 2000). However, the results reported for the relationship between cortisol and aggression are inconsistent. Although there is a large body of work which reports a negative relationship between cortisol levels and aggression (e.g. McBurnett et al., 2000), there are also several studies which have observed a positive association between these two variables (e.g. Azurmendi et al., 2016; Barzman et al., 2013).

In relation to establishing prototypes or profiles associated with aggressive behavior, it should be noted that, while psychological clusters have been established in association with this behavior (e.g. Belgrave, Nguyen, Johnson, \& Hood, 2011), psychobiological clusters have yet to be determined. To the best of our knowledge, hardly any studies have established clusters integrating both hormone and psychological variables in association with aggression, either in adults or children, as part of an attempt to identify the different profiles which may underlie different aggression levels in the subjects studied.

One of the main arguments supporting the need to study those psychobiological clusters potentially associated with aggressive behavior is linked to recent evidence of an interaction between psychological and biological variables for explaining aggressive behavior, as well as to the existence of associations between psychological variables and hormones. Sánchez-Martín et al. (2011) found interactive effects of anger and androstenedione on aggression in boys, and PascualSagastizabal et al. (2014) also found that testosterone moderated the relationship between maternal authoritarian parenting style and aggressive behavior in boys and girls. Furthermore, evidence has also been found of an association between the psychological variables (anger and empathy) and hormones (testosterone and cortisol) analyzed in the present study. Thus, diverse studies have reported a positive association between high testosterone levels and anger measures (Hohlagschwandtner, Husslein, Klier, \& Ulm, 2001; Persky, Smith, \& Basu, 1971; van Honk et al., 2000; van Honk et al., 1999; Wirth \& Schultheiss, 2007), while many others have found a negative relationship between testosterone and empathic capacity (Auyeung et al., 2009; Chapman et al., 2006; Hermans, Putman, \& van Honk, 2006; Pascual-Sagastizabal et al., 2013; van Honk et al., 2011). Few studies, however, have focused on the relationship between cortisol and anger and cortisol and empathy. While some evidence has been found of a positive association between cortisol and empathy measures (Nakayama et al., 2007; Zilioli et al., 2015), the findings linked to cortisol levels and anger measures are inconsistent, with some studies finding a positive association (Putman, Hermans, \& van Honk, 2007), others a negative one (Lerner, Dahl, Hariri, \& Taylor, 2007) and others no relationship at all (Lewis \& Ramsay, 2005). Together, these findings support the idea of psychobiological clusters associated with different levels of aggression in children.

The approach proposed in our study, which combines both hormone and psychological variables, enables us to analyze the biological and psychological components of a holistic (psychobiological) system. In light of evidence showing that some psychological variables (anger) foster children's aggressive behavior while others (empathy) inhibit it, and given that certain levels of some hormones have been associated with an increase in aggression, our aim here was to analyze the possible existence of psychobiological clusters in which specific levels (low or high) of psychological variables, alongside specific levels (low or high) of hormones, form a cluster characterized by a certain level of aggressive behavior. If, moreover, we also take into account the moderating effects described above (such as, for example, that of hormones on psychological variables) and the direct association between hormones and psychological variables, it seems only logical to search for the psychobiological markers of 
aggressive behavior, since their early detection would facilitate both prevention and interaction. Although analyzing the influence of psychological and biological variables separately serves to notably increase our knowledge of the mechanisms underlying aggression, their joint analysis, in the form of psychobiological profiles or markers, will provide even greater insight into the complex psychobiological mechanisms involved, and will therefore shed light, from an applied perspective, on how to prevent or mitigate this behavior, which has so many negative consequences at both an individual and social level.

In this study, we specifically expected the cluster containing the most aggressive subjects to also contain higher measures of anger, testosterone and cortisol, and lower measures of empathy. Moreover, we expected the clusters containing the least aggressive subjects to also contain lower measures of anger, testosterone and cortisol, and higher measures of empathy.

\section{Method}

\section{Participants}

The study sample comprised 139 eight-year-old children ( 80 boys and 59 girls) from a city in the north of Spain. Several authors have suggested that the transition between early and middle childhood is a "sensitive window" that is vital to the configuration of specific behavioral strategies, due to both children's susceptibility to the effects of their environment and the plasticity potentially induced by hormones during this period (Del Giudice, Angeleri, \& Manera, 2009; Del Giudice et al., 2018). During this childhood transition period, which takes place between the ages of 6 and 8 years, children engage in increased social activities with their peers and begin to complete effectively for dominance in group hierarchies. Richard Tremblay, one of the world's leading experts in childhood aggression, argues that from this stage onwards (8 years), it is increasingly difficult to implement successful intervention strategies for combating aggressive behavior (Tremblay, 2008). The socioeconomic status of the sample was considered medium and medium-high, based on our knowledge of the area in which participants live. Of the 4 participating schools, 3 were public ones and the fourth one was a semi-private one (i.e. a private school that receives some state funding), a ratio which reflects the predominance of public schools in the region. Moreover, all schools were co-ed (i.e. the student body was made up of both boys and girls). At the beginning of the academic year, a letter was sent to 201 families providing them with detailed information about the study. The letter also contained a document which they were asked to sign in order to give or refuse their consent for their child to participate in the study. Of the 201 families contacted, 165 gave their consent. Finally, for the statistical analyses, the sample group was reduced to 139 due to incomplete data (child being ill on the day of the test, families failing to return their questionnaires, etc.). None of the tests used during this study were invasive and all were carried out in the school itself; the project was approved by the ethics committee at the institution to which the authors belong.

\section{Aggressive behavior measure}

Aggressive behavior was assessed using the Spanish version of the Direct and Indirect Aggression Scale (DIAS) (Björkqvist \& Österman, 1998), a peer-rating measurement instrument for aggressive behavior. Previous studies have shown that this test is a useful instrument for measuring different types of aggression in young children (e.g. Valles \& Knutson, 2008). The DIAS comprises 24 items (e.g. Hits the other one?, Shuts the other one out of the group?) in which each child is asked to rate each of their same-sex classmates for behaviors linked to aggression on a 5-point Likerttype scale, ranging from 0 (never) to 4 (very often). More precisely, they rate behavior linked to physical aggression (7 items), verbal aggression (5 items) and indirect aggression (12 items).

In order to facilitate the collective administration of the test, the questions were asked in table format, thus allowing the participants to assess all their same-sex classmates in every question.

This test resulted in a total aggression measurement based on the scores obtained by participants in the three sub-scales (physical, verbal and indirect aggression), since the correlations between them were over $r=.94$. The reliability coefficient obtained in this questionnaire was Cronbach's alpha $=.97$.

\section{Empathy measure}

The test used to measure empathy was the EQ-C (Empathy Quotient-Child Version), adapted for children by Chapman et al. (2006) from the adult EQ instrument developed by Baron-Cohen and Wheelwright (2004).

The test was administered to parents, who were asked to assess their child's capacity for empathy. The questionnaire contains 27 items with four response options and three possible scores which are interpreted as follows: "definitely agree $=2$ ", "slightly agree $=1$ ", "slightly disagree $=1$ " and "definitely disagree $=0$ ". Examples of items include "My child likes to look after other people" and "My child would worry about how another child would feel if they weren't invited to a party". The maximum score that can be obtained in this 
test is 54 . The reliability coefficient obtained in our sample was Cronbach's alpha $=.78$.

\section{Anger measure}

Anger was measured using the STAXI-NA (StateTrait Anger Expression Inventory for Children and Adolescents, TEA), developed by del Barrio, Aluja, and Spielberger (2004) for children aged between 8 and 17. This is a self-reporting test that assesses different components (Experience, Expression and Control) and facets (State and Trait) of anger through 32 items rated on a 3-point Likert-type scale (almost never $=1$, sometimes $=2$ and almost always $=3$ ). Given the aims of the study, we decided to use the trait anger subscale (8 items), since it refers to children's tendency to have more frequent and intense angry reactions (e.g. "I'm easily irritated" and "I lose my temper easily"). The reliability coefficient of the trait anger sub-scale was Cronbach's alpha $=.71$.

\section{Determination of salivary hormone levels}

Two saliva samples were collected from each child by trained personnel in the classrooms on weekdays. Samples were collected at $09.00 \mathrm{hr}$, before lessons started, in order to avoid concentration changes due to diurnal hormonal fluctuations. Participants were asked to deposit a saliva sample into a plastic cup (sterile container for the hygienic collection of biological samples) by passive drool. To help them salivate they were offered a sweet on the condition that they were not allowed to eat it until they had given a large enough sample. The aim was to stimulate saliva production through the promise of an appetizing reward. Samples were frozen and stored in the laboratory at $-80^{\circ} \mathrm{C}$ until analysis. On the day of the analysis, the samples were centrifuged at 3,000 rpm for $15 \mathrm{~min}$ to remove mucins. Both of the samples gathered from each subject were assayed in duplicate. The average of each duplicate test was used in the analyses. All samples were assayed using an enzyme immunoassay kit (Salimetrics, State Collage, USA). For hormone levels, the average intra-assay coefficient of variation $(\mathrm{CV})$ was less than $3 \%$ and the inter-assay coefficient of variation $(\mathrm{CV})$ was less than $14 \%$, calculated on the basis of control samples.

\section{Statistical analysis}

All the variables were transformed into $Z$ scores in order to cancel the effect of range disparity problems. Since they did not have a normal distribution, aggressive behavior, hormone levels, empathy and anger measures were normalized using the Bloom transformation, which is the most common inverse normal transformation (Maritz, 1982). The analysis of the correlations observed between the different variables studied is shown in Table 1. Analyses of variance (ANOVAs) were performed to analyze possible sex differences in the variables. The results revealed that girls scored higher in empathy, $F(1,137)=14.36$; $p=.0001 ; d=0.66$, than boys. No statistically significant differences were found between boys and girls in any of the other variables.

A cluster analysis was carried out using the hierarchical-clustering subroutine to cluster participants in accordance with their biological and psychological characteristics. A multivariate discriminant analysis was performed to investigate the integrity of those clusters derived from the cluster analysis. Finally an ANOVA was carried out to determine differences in aggressive behavior between clusters.

All the analyses were carried out using the IBM SPSS 21.00 statistical package.

\section{Results}

\section{Clusters based on psychological and biological variables}

With the aim of exploring profile differences among 8-year-old children, a Cluster Analysis was performed in accordance with biological (cortisol and testosterone levels) and psychological (empathy and trait anger) measures, in order to analyze possible disparities in terms of aggressive behavior. Ward's clustering method was applied, in which similarities between each pair of subjects are measured in accordance with the squared Euclidian distance. A cut-off criterion was

Table 1. Correlations between Aggression, Testosterone, Cortisol, Anger and Empathy

\begin{tabular}{|c|c|c|c|c|c|}
\hline & Testosterone & Cortisol & Aggression & Anger & Empathy \\
\hline Testosterone & 1 & & & & \\
\hline Cortisol & $.459^{* *}$ & 1 & & & \\
\hline Aggression & .089 & $.236^{* *}$ & 1 & & \\
\hline Anger & -.010 & .030 & $.336^{* *}$ & 1 & \\
\hline Empathy & -.022 & -.047 & $-.321^{* *}$ & $-.170^{*}$ & 1 \\
\hline
\end{tabular}

Note: ${ }^{* *} p<.01 .{ }^{*} p<.05$. 
applied to the dendogram obtained using this clustering method in order to select the main clusters, with the cut-off point being equal to 8 . Three clusters were thus obtained.

To study and confirm the goodness of fit of the clusters obtained, a discriminant analysis was conducted using Wilk's Lambda step-by-step inclusion method (Figure 1). The discriminant model applied accounted for $100 \%$ of cases obtained by the cluster solution, confirming the statistical validity of these clusters, as well as their psychobiological description. The cluster analysis was then conducted once again with the psychological and biological variables included in the discriminant model.

As shown in Table 2, this analysis revealed three clusters which were clearly different as regards their biological and psychological characteristics:

Cluster 1 ( $n=51,28$ boys and 23 girls): Subjects with higher anger levels, lower empathy levels and higher testosterone and cortisol levels.

Cluster 2 ( $n=48,25$ boys and 23 girls): Subjects with moderate anger levels, moderate empathy levels and lower testosterone and cortisol levels.

Cluster 3 ( $n=40,27$ boys and 13 girls): Subjects with lower anger levels, higher empathy levels and higher testosterone and cortisol levels.

A one-way ANOVA and Tuckey's post-hoc test were conducted to determine whether the three clusters found were significantly different as regards both hormone and psychological variables. The results revealed statistically significant differences between the clusters in relation to both kinds of variable: testosterone, $F(2,136)=50.51, M S E=27.97, p<.0001, \eta^{2}=.43$; cortisol, $F(2,136)=45.27, M S E=26.59, p<.0001, \eta^{2}=.40$; empathy, $F(2,136)=22.05, M S E=27.98, p<.0001$, $\eta^{2}=.45 ;$ and anger, $F(2,136)=56.31, M S E=16.08$, $p<.0001, \eta^{2}=.24$. The post-hoc analysis revealed statistical differences between clusters in empathy and anger and in relation to hormones. The results showed that Cluster 2 was significantly different from Clusters 1 and 3 (Table 2 and Figure 1).

\section{Differences in Aggressive Behavior}

A one-way ANOVA and Tuckey's post-hoc test were conducted to determine whether or not the different psychobiological clusters found were associated with differences in aggressive behavior. The results revealed statistically significant differences between the clusters in relation to aggressive behavior, $F(2,136)=15.92$, $M S E=12.87, p<.0001, \eta^{2}=.19$. The post-hoc analysis revealed that the children in Cluster 1 , characterized by having higher levels of anger, lower levels of empathy and higher levels of testosterone and cortisol, were more aggressive than their counterparts in the other two clusters (Figure 2).

\section{Discussion}

The results of this study suggest that there are psychobiological clusters associated with aggressive behavior in school-aged children. The data analysis divided the sample group of 8-year-old children into three homogeneous clusters based on 4 variables, two psychological ones (anger and empathy) and two biological ones (testosterone and cortisol). The analysis of variance (ANOVA) revealed that the cluster characterized by having higher anger levels, lower empathy levels and higher testosterone and cortisol levels (Cluster 1) was

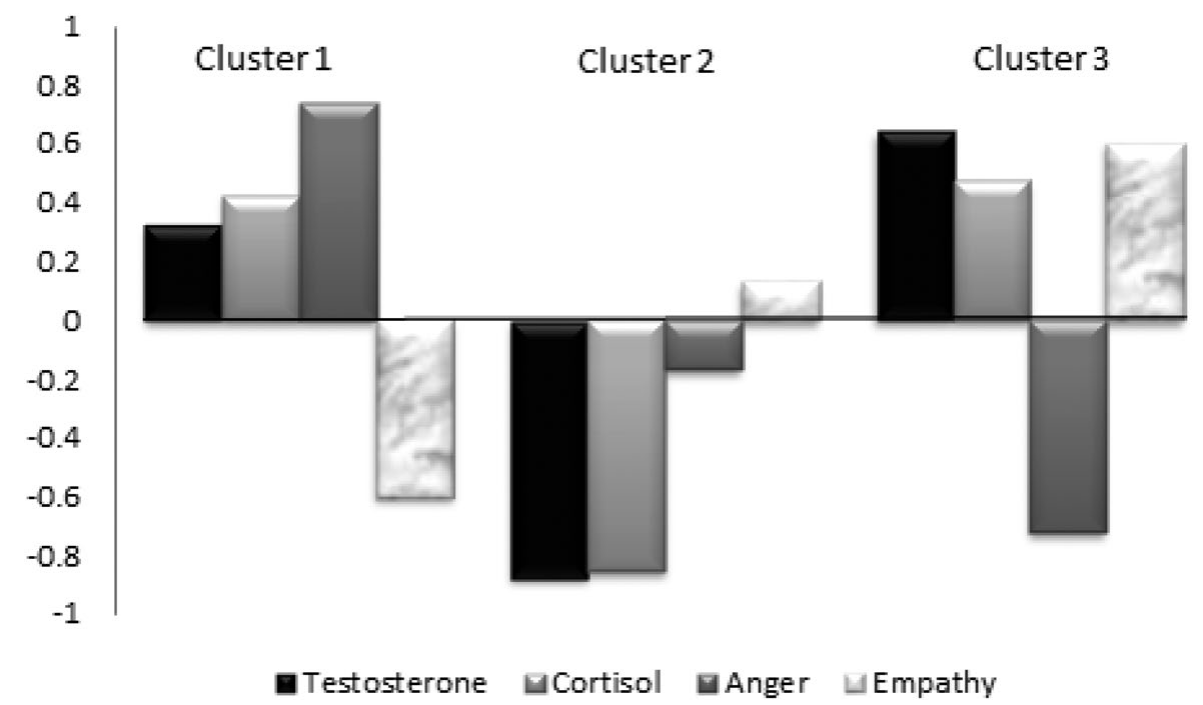

Figure 1. Final cluster with the four variables included in the discriminant model (Testosterone, Cortisol, Empathy and Anger) 
Table 2. Descriptive Statistics for the Different Clusters and post hoc Analysis

\begin{tabular}{lcrc}
\hline & $\begin{array}{l}\text { Cluster } 1^{*} \\
N=54\end{array}$ & \multicolumn{1}{l}{$\begin{array}{l}\text { Cluster } 2 \ddagger \\
N=48\end{array}$} & \multicolumn{1}{l}{$\begin{array}{l}\text { Cluster } 3+ \\
N=40\end{array}$} \\
\hline Anger & $.75 \pm .71 \ddagger \dagger$ & $-.17 \pm .84^{*} \dagger$ & $-.72 \pm .60^{*} \ddagger$ \\
Empahty & $-.60 \pm .75 \ddagger \dagger$ & $.14 \pm .95^{*} \dagger$ & $.61 \pm .82^{*} \ddagger$ \\
Testosterone & $.32 \pm .83 \ddagger$ & $-.88 \pm .65^{*} \dagger$ & $.65 \pm .68 \ddagger$ \\
Cortisol & $.43 \pm .81 \ddagger$ & $-.86 \pm .72^{*} \dagger$ & $.48 \pm .75 \ddagger$ \\
\hline
\end{tabular}

Note: Post hoc comparisons were made using the Tuckey HSD test.

*Shows significant differences with the Cluster 1. ‡Shows significant differences with the Cluster 2. tShows significant differences with the Cluster 3.

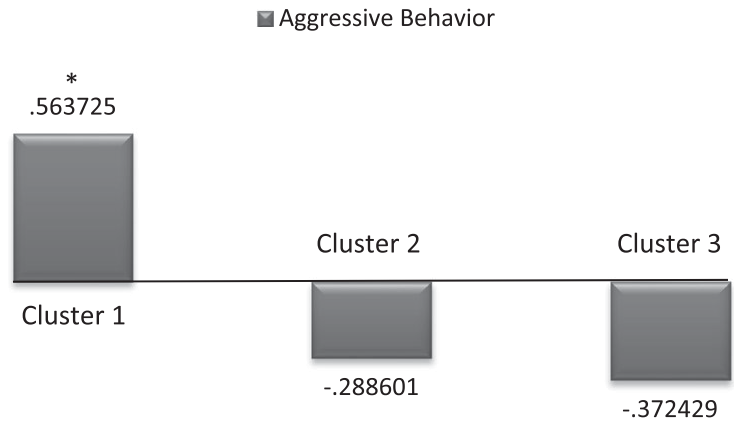

Figure 2. Post-hoc analyses for comparing aggressive behavior between clusters

more aggressive than the other two. This result is consistent with that reported in the literature, since although no studies on aggressive behavior to date have focused on both psychological and biological variables at the same time (in a cluster configuration), those that have analyzed psychological variables (anger and empathy) have found a negative association between empathy and aggression, and a positive relationship between anger and aggression (Fung et al., 2015; Strayer \& Roberts, 2004). It is logical to assume that subjects with higher anger levels also have lower empathy levels and are therefore more aggressive, as the result of a kind of accumulation of different psychological factors. In addition to the studies by Fung et al. (2015) and Strayer and Roberts (2004), which have reported consistent findings in this sense, a cluster analysis conducted by Belgrave et al. (2011) found that the "well-adjusted" prototype (11-14 years old) scored higher than the mean in attributes such as empathy and anger management, while the "poorlyadjusted" prototype scored below average in the same attributes.

The most aggressive cluster was found to contain subjects with relatively high levels of cortisol and testosterone. Nevertheless, we also found that subjects with higher levels of these hormones were split between two clusters (Cluster 1 and Cluster 3), which were associated with different levels of aggression (high and low, respectively) and had different levels of empathy and anger. Thus, subjects with relatively high testosterone and cortisol levels were found to be more aggressive when these biological variables were accompanied by higher anger and lower empathy scores (Cluster 1), and less aggressive when subjects had lower anger and higher empathy levels (Cluster 3). There was also another well-defined cluster that was characterized by low aggression levels, in which subjects had lower levels of both testosterone and cortisol, and moderate anger and empathy levels (Cluster 2).

Prior research has found a positive association between various steroid hormones (testosterone and cortisol) and aggressive behavior in children across different age groups (Azurmendi et al., 2016; Lerner et al., 2007; Sánchez-Martín et al., 2000), a finding which has prompted some authors to postulate that said hormones contribute to the development and maintenance of aggression in school-aged children. Nevertheless, the results of this present study seem to demonstrate that it is psychological factors (anger and empathy) that best define or discriminate the psychobiological profile linked to aggressive behavior, more so than hormone ones, since under equal biological conditions (higher testosterone and cortisol levels), subjects with higher levels of anger and lower levels of empathy were found to be more aggressive. There are two possible ways of interpreting these results: a) it may be that the potential facilitating effect of high testosterone and cortisol levels on aggression is further enhanced by high levels of anger and low levels of empathy (Cluster 1) and attenuated by low levels of anger and high levels of empathy (Cluster 3 ); b) or alternatively, it may be that the potential facilitating effect of high anger and low empathy levels on aggression is further enhanced by high testosterone and cortisol levels (Cluster 1), and the attenuating effect of low anger and high empathy levels is not affected by higher levels of testosterone and cortisol (Cluster 3). It should not be forgotten that sex-differentiated profiles may also exist in which hormone levels would be different for boys and girls. However, due to the limited size of our sample we were unable to verify this.

Two possible models have been suggested to explain the association between low empathy and high anger scores and high aggression levels (Strayer \& Roberts, 2004). The first is a "main effects" model, in which a greater understanding of others (translated into empathy) at both a cognitive and emotional level brings about a decrease in both anger and aggression. The second is a "mediated model", in which empathy decreases aggression levels by decreasing anger levels. 
Given that a number of studies have associated anger with testosterone (e.g. Wirth \& Schultheiss, 2007) and cortisol (e.g. Rausch et al., 2015), and others have also found a relationship between empathy and testosterone (e.g. Pascual-Sagastizabal et al., 2013) and cortisol (e.g. Wolf et al., 2015), it would be interesting in the future to consider integrating all these factors into one of the two models outlined above.

Nevertheless, our study does have some limitations which should not be overlooked. Firstly, it is important to clarify that the results found cannot be generalized, since the study sample is not representative. Secondly, only two saliva samples were collected, on different days, for the hormone measures. Finally, measuring other androgens, such as androstenedione, may have enriched the analyses at a biological level.

To conclude, we would like to point out that no studies to date have analyzed aggressive behavior from a psychobiological perspective, using clustering based on both biological and psychological characteristics. Our study seems to indicate that higher or lower levels of the psychological variables studied here (anger and empathy) are better able to define the prototype associated with more aggressive behavior than higher or lower levels of the biological variables analyzed (testosterone and cortisol). Detecting profiles of this nature in early development phases may provide useful insight into the psychobiological configuration underlying the development of aggressive behavior, which may subsequently give rise to severe maladjustment in an individual's later psychosocial development. It may also be useful for implementing prevention strategies based on a more thorough knowledge of our organism's holistic functioning. Thus, the perspective and approach adopted in this study are innovative and provide interesting information for the study of aggressive behavior during childhood.

\section{References}

Archer J. (2006). Testosterone and human aggression: An evaluation of the challenge hypothesis. Neuroscience and Biobehavioral Reviews, 30, 319-345. https:/ / doi.org/ 10.1016/j.neubiorev.2004.12.007

Archer J. (2009). The nature of human aggression. International Journal of Law and Psychiatry, 32, 202-208. https://doi.org/10.1016/j.ijlp.2009.04.001

Auyeung B., Baron-Cohen S., Ashwin E., Knickmeyer R., Taylor K., \& Hackett G. (2009). Fetal testosterone and autistic traits. British Journal of Psychology, 100, 1-22. https://doi.org/10.1348/000712608X311731

Azurmendi A., Pascual-Sagastizabal E., Vergara A. I., Muñoz J. M., Braza P., Carreras R., ... Sánchez-Martin J. R. (2016). Developmental trajectories of aggressive behavior in children from ages 8 to 10: The role of sex and hormones. American Journal of Human Biology, 28, 90-97. https://doi.org/10.1002/ajhb.22750
Baron-Cohen S., \& Wheelwright S. (2004). The empathy quotient: An investigation of adults with Asperger syndrome or high functioning autism, and normal sex differences. Journal of Autism and Developmental Disorders, 34, 163-175. https://doi.org/10.1023/ B:JADD.0000022607.19833.00

Barzman D. H., Mossman D., Appel K., Blom T. J., Strawn J. R., Ekhator N. N., ... \& Geracioti T. D., Jr. (2013). The association between salivary hormone levels and children's inpatient aggression: A pilot study. Psychiatric Quarterly, 84, 475-484. https://doi.org/ 10.1007/s11126-013-9260-8

Belgrave F. Z., Nguyen A. B., Johnson J. L., \& Hood K. (2011). Who is likely to help and hurt? Profiles of African American adolescents with prosocial and aggressive behavior. Journal of Youth and Adolescence, 40, 1012-1024. https://doi.org/10.1007/s10964-010-9608-4

Berger C., Batanova M., \& Duncan C. J. (2015). Aggressive and prosocial? Examining latent profiles of behavior, social status, machiavellianism, and empathy. Journal of Youth and Adolescence, 44, 2230-2244. https://doi.org/10.1007/ s10964-015-0298-9

Björkqvist K., \& Österman K. (1998). Scales for research on interpersonal relations. Vasa, Finland: Abo Akademi University.

Campbell A. (2006). Sex differences in direct aggression: What are the psychological mediators? Aggression and Violent Behavior, 11, 237-264. https:/ / doi.org/10.1016/ j.avb.2005.09.002

Clay D. L., Hagglund K. J., Kashani J. H., \& Frank R. G. (1996). Sex differences in anger expression, depressed mood, and aggression in children and adolescents. Journal of Clinical Psychology in Medical Settings, 3, 79-92. https:/ / doi.org/10.1007/BF01989291

Côté S. M., Vaillancourt T., Barker E. D., Nagin D., \& Tremblay R. E. (2007). The joint development of physical and indirect aggression: Predictors of continuity and change during childhood. Development and Psychopathology, 19, 37-55. https://doi.org/10.1017/S0954579407070034

Chapman E., Baron-Cohen S., Auyeung B., Knickmeyer R., Taylor K., \& Hackett G. (2006). Fetal testosterone and empathy: Evidence from the Empathy Quotient (EQ) and the "Reading the Mind in the Eyes" Test. Social Neuroscience, 1, 135-148. https://doi.org/10.1080/ 17470910600992239

Daff E. S., Gilbert F., \& Daffern M. (2015). The relationship between anger and aggressive script rehearsal in an offender population. Psychiatry Psychology and Law, 22, 731-739. https://doi.org/10.1080/13218719.2014.986837

del Barrio V., Aluja A., \& Spielberger C. (2004). Anger assessment with the STAXI-CA: Psychometric properties of a new instrument for children and adolescents. Personality and Individual Differences, 37, 227-244. https:/ / doi.org/10.1016/j.paid.2003.08.014

Del Giudice M., Angeleri R., \& Manera V. (2009). The juvenile transition: A developmental switch point in human life history. Developmental Review, 29, 1-31. https:/ / doi.org/10.1016/j.dr.2008.09.001

Del Giudice M., Barrett E. S., Belsky J., Hartman S., Martel M. M., Sangenstedt S., \& Kuzawa C. W. (2018). 
Individual differences in developmental plasticity: A role for early androgens? Psychoneuroendocrinology, 90, 165-173. https://doi.org/10.1016/j.psyneuen.2018.02.025

Feshbach N. D. (1975). Empathy in children: Some theoretical and empirical considerations. The Counseling Psychologist, 5, 25-30. https:/ / doi.org/10.1177/ 001100007500500207

Fung A. L. C., Gerstein L. H., Chan Y., \& Engebretson J. (2015). Relationship of aggression to anxiety, depression, anger, and empathy in Hong Kong. Journal of Child and Family Studies, 24, 821-831. https://doi.org/10.1007/ s10826-013-9892-1

Hermans E. J., Putman P., \& van Honk J. (2006). Testosterone administration reduces empathetic behavior: A facial mimicry study. Psychoneuroendocrinology, 31(7), 859-866. https://doi.org/10.1016/j.psyneuen.2006.04.002

Hohlagschwandtner M., Husslein P., Klier C., \& Ulm B. (2001). Correlation between serum testosterone levels and peripartal mood states. Acta Obstetricia et Gynecologica Scandinavica, 80(4), 326-330. https://doi.org/10.1034/ j.1600-0412.2001.080004326.x

Jolliffe D., \& Farrington D. P. (2006). Examining the relationship between low empathy and bullying. Aggressive Behavior, 32, 540-550. https:/ / doi.org/ 10.1002/ab.20154

Leiberg S., \& Anders S. (2006). The multiple facets of empathy: A survey of theory and evidence. Progress in Brain Research, 156, 419-440. https: / /doi.org/10.1016/ S0079-6123(06)56023-6

Lerner J. S., Dahl R. E., Hariri A. R., \& Taylor S. E. (2007). Facial expressions of emotion reveal neuroendocrine and cardiovascular stress responses. Biological Psychiatry, 61, 253-260. https: / / doi.org/10.1016/j.biopsych.2006.08.016

Lewis M., \& Ramsay D. (2005). Infant emotional and cortisol responses to goal blockage. Child Development, 76(2), 518-530. https:/ / doi.org/10.1111/j.1467-8624.2005.00860.x

Maritz J. S. (1982). Distribution free statistical methods. London, UK: Chapman and Hall.

McBurnett K., Kumar A. M., Kumar M., Perez D., Lahey B. B., \& Shaw J. A. (2000). Aggression, anxiety, and salivary cortisol in child psychiatry inpatients. Biological Psychiatry, 47(8, Suppl. 1), S150-S151. https:/ / doi.org/10.1016/S0006-3223(00)00765-4

Nakayama Y., Takahashi T., Wakabayashi A., Ono H., \& Radford M. H. B. (2007). Sex differences in the relationship between cortisol levels and the empathy and systemizing quotients in humans. Neuro Endocrinology Letters, 28(4), 445-448.

Pascual-Sagastizabal E., Azurmendi A., Sánchez-Martín J. R., Braza F., Carreras M. R., Muñoz J. M., \& Braza P. (2013). Empathy, estradiol an androgen levels in 9-year-old children. Personality and Individual Differences, 54, 936-940. https://doi.org/10.1016/j.paid.2013.01.019

Pascual-Sagastizabal E., Azurmendi A., Braza F., Vergara A. I., Cardas J., \& Sánchez-Martín J. R. (2014). Parenting styles and hormone levels as predictors of physical and indirect aggression in boys and girls. Aggressive Behavior, 40, 465-473. https://doi.org/10.1002/ab.21539

Persky H., Smith K. D., \& Basu G. K. (1971). Relation of psychologic measures of aggression and hostility to testosterone production in man. Psychosomatic Medicine, 33(3), 265-267. https://doi.org/10.1097/00006842197105000-00007

Putman P., Hermans E. J., \& van Honk J. (2007). Exogenous cortisol shifts a motivated bias from fear to anger in spatial working memory for facial expressions. Psychoneuroendocrinology, 32(1), 14-21. https://doi. org/10.1016/j.psyneuen.2006.09.010

Rausch J., Gäbel A., Nagy K., Kleindienst N., Herpertz S. C., \& Bertsch K. (2015). Increased testosterone levels and cortisol awakening responses in patients with borderline personality disorder: Gender and trait aggressiveness matter. Psychoneuroendocrinology, 55, 116-127. https://doi.org/10.1016/j.psyneuen.2015.02.002

Sánchez-Martín J. R., Fano E., Ahedo L., Cardas J., Brain P. F., \& Azpíroz A. (2000). Relating testosterone levels and free play social behavior in male and female preschool children. Psychoneuroendocrinology, 25, 773-783. https://doi.org/10.1016/S0306-4530(00)00025-1

Sánchez-Martín J. R., Azurmendi A., Pascual-Sagastizabal E., Cardas J., Braza F., Braza P., ... Muñoz J. M. (2011). Androgen levels and anger and impulsivity measures as predictors of physical, verbal and indirect aggression in boys and girls. Psychoneuroendocrinology, 36(5), 750-760. https://doi.org/10.1016/j.psyneuen.2010.10.011

Strayer J., \& Roberts W. (2004). Empathy and observed anger and aggression in five-year-olds. Social Development, 13, 1-13. https://doi.org/10.1111/ j.1467-9507.2004.00254.x

Tremblay R. E. (Ed.) (2008). Prèvenir la violence dès la petite enfance [Preventing violence from infancy]. Paris, France: Odile Jacob.

Tremblay R. E., Vitaro F., \& Côté S. M. (2018). Developmental origins of chronic physical aggression: A bio-psico-social model for the next generation of preventive interventions. Annual Review of Psychology, 69, 383-407.https:/ / doi. org/10.1146/annurev-psych-010416-044030

Valles N.-L., \& Knutson J. F. (2008). Contingent responses of mothers and peers to indirect and direct aggression in preschool and school-aged children. Aggressive Behavior, 34, 497-510. https://doi.org/10.1002/ab.20268

van Honk J., Schutter D. J., Bos P. A., Kruijt A.-W., Lentjes E. G., \& Baron-Cohen S. (2011). Testosterone administration impairs cognitive empathy in women depending on second-to-fourth digit ratio. Proceedings of the National Academy of Sciences of the United States of America, 108(8), 3448-3452. https:/ /doi.org/10.1073/ pnas. 1011891108

van Honk J., Tuiten A., van den Hout M., Koppeschaar H., Thijssen J., de Haan E., \& Verbaten R. (2000). Conscious and preconscious selective attention to social threat: Different neuroendocrine response patterns. Psychoneuroendocrinology, 25(6), 577-591. https: / / doi. org/10.1016/S0306-4530(00)00011-1

van Honk J., Tuiten A., Verbaten R., van den Hout M., Koppeschaar H., Thijssen J., \& de Haan E. (1999). Correlations among salivary testosterone, mood, and selective attention to threat in humans. Hormones and Behavior, 36, 17-24. https://doi.org/10.1006/hbeh. 1999.1521 
Warden D., \& MacKinnon S. (2003). Prosocial children, bullies and victims: An investigation of their sociometric status, empathy and social problemsolving strategies. British Journal of Developmental Psychology, 21, 367-385. https:/ / doi.org/10.1348/ 026151003322277757

Wirth M. M., \& Schultheiss O. C. (2007). Basal testosterone moderates responses to anger faces in humans. Physiology \& Behavior, 90, 496-505. https:/ / doi. org/10.1016/j.physbeh.2006.10.016
Wolf O. T., Schulte J. M., Drimalla H., Hamacher-Dang T. C., Knoch D., \& Dziobek I. (2015). Enhanced emotional empathy after psychosocial stress in young healthy men. Stress, 18, 631-637. https:/ /doi.org/10.3109/10253890. 2015.1078787

Zilioli S., Ponzi D., Henry A., \& Maestripieri D. (2015). Testosterone, cortisol and empathy: Evidence for the dual-hormone hypothesis. Adaptive Human Behavior $\mathcal{E}$ Physiology, 1, 421-433. https://doi.org/10.1007/s40750014-0017-x 